\title{
The Reg 'Activ Cholesterol Positive Effects in Asymptomatic Volunteers with Borderline Values of Cardio-Metabolic Risk Factors are Sustainable in Long-Term Run
}

\author{
Tiiu Kullisaar* \\ Department of Biochemistry, Institute of Biomedicine and Translational Medicine, Faculty of Medicine, University of Tartu, Tartu, Estonia \\ ${ }^{\star}$ Corresponding Author: Dr. Tiiu Kullisaar, PhD, Department of Biochemistry, Institute of Biomedicine and Translational Medicine, Faculty of Medicine, University of Tartu, \\ 19 Ravila Street, 50411, Tartu, Estonia; Tel: +372 737 4314; Email: tiiu.kullisaar@ut.ee
}

Received: February 04, 2019; Accepted: February 05, 2019; Published: February 22, 2019;

\begin{abstract}
Previous part of clinical trial (ISRCTN55339917) highlighted several beneficial effects of RAC (Reg' Activ Cholesterol ${ }^{\mathrm{TM}}$ ) comprising also antioxidative and antiatherogenic probiotic Lactobacillus fermentum ME-3[1]. Cardiovascular diseases are number one cause of death globally. Beneficial modifying of the cardio-metabolic profile has major impact for long-term success. Our previous open-label pilot study showed a complex positive influence of a food supplement Reg'Activ Cholesterol (RAC) on Cardio-Metabolic Parameters (CMP).
\end{abstract}

To test in very long-term run both sustainability of these effects and safety of RAC we now aimed to investigate RAC effects during 3 years. Main randomizing sign was prediabetic level of glycated haemoglobin (HbA1c between 5.7-6.4\%) in clinically asymptomatic volunteers who had elevated level of low density lipoprotein cholesterol (LDL-chl) and oxidized LDL (oxLDL).

The level of Cholesterol, LDL-cholesterol and oxLDL and HbA1c\% decreased significantly and HDL-cholesterol increased significantly.

RAC has shown a complex positive effect on cardiovascular risk profile. Still investigations are needed to evaluate its long-term effects on clinical outcomes. Trial registration: ISRCTN55339917 http://www.isrctn.com/ISRCTN55339917

Keywords: Reg' Activ Cholesterol ${ }^{\mathrm{Tm}}$ Lactobacillus fermentum ME-3, pre-diabetes, nutriceuticals

\section{Introduction}

The study presented here is a sub study from a larger set of investigations to evaluate the effect of a food supplement Reg'Activ Cholesterol (RAC) produced by VF Bioscience Company to cardiometabolic risk profile. Our previous open-label pilot study [1] showed a complex positive influence of RAC on Cardio-Metabolic Parameters (CMP). Cardiovascular Diseases (CVD) and its complications still hold primacy of health care for societies despite decades of investigations, guidelines of primary and secondary prevention and a long list of medicines used to fight them. Managing atherosclerosis, the patho-physiological process underlying CVD, still needs a lot of improvement starting from finding a good set of diagnostic markers until blocking the process that culminates in total vascular obstruction. For decades we have known that dyslipidemia and especially an elevated LDL-cholesterol are linked to the pathogenesis of CVD. The acknowledgement of the role of LDL oxidation, tightly associated with inflammation, is not new either [2]. Diabetes often co-exists with CVD and if present it complicates the course of CVD. The pathophysiological phenomena oxidative stress (OxS), chronic inflammation and glycation cause cardio-metabolic changes that are common in the development of metabolic syndrome, diabetes and CVD [3].The ATTICA study showed that a permanent low- grade/ high-normal inflammation (LGI) and an advanced glycation both may enhance the development of CVD thus linking LGI and diabetes [4]. It is accepted that the ratio of blood triglycerides to HDL cholesterol (HDL-cholesterol) (TG/HDL-cholesterol) describes the insulin receptor resistance [5]. A possible role of a poor or unbalanced intestinal microbiota in atherosclerosis pathogenesis has also been described [6]. Thus interventions that favorably modify the cardiometabolic profile may find a place in a comprehensive long-term strategy both in CVD and prediabetes /diabetes management.

RAC is a complex designed of components that theoretically could positively affect $\mathrm{OxS}$ and LDL oxidation and/or glycation and/or other CMP and inflammatory markers.

The components of RAC are: red yeast rice, ubiquinol, vitamin E, vitamins B6, B9, B12 and Lactobacillus fermentum ME-3[1].

Lactobacillus fermentum ME-3 (DSM 14241) (LFME-3), a component in RAC is a strain isolated from a healthy 1-year old child [7]. The strain has both antimicrobial and antioxidative functional properties [8-10]. Several clinical studies [11-14] have shown health benefits of fermented products containing LFME-3. Our previous open-label pilot study [1] showed a complex positive influence of a new innovative food supplement RAC on CMP. 
Tiiu Kullisaar (2019) The Reg 'Activ Cholesterol Positive Effects in Asymptomatic Volunteers with Borderline Values of Cardio-Metabolic Risk Factors are Sustainable in Long-Term Run

The aim of the study was to find out if a compiled probiotic containing formula RAC (VF Bioscience) could have a clinically significant positive effect on cardiovascular disease risk factor profiles in clinically asymptomatic volunteers with borderlinehigh values of CMPs (alike) (primary targets were LDL-cholesterol, oxLDL and $\mathrm{HbA1c} \%$ ). Thus, targeted supplemental intervention that favorably modifies the cardio-metabolic profile may have impact for adjuvant long-term strategy both in CVD and pre-diabetes/diabetes management.

\section{Patients and Methods}

After finishing the first phase of clinical trial (ISRCTN55339917) [1] five clinically healthy volunteers with borderline values of risk factors for CVD and pre-diabetes sign were asked to continue this open label trial for 3 years, from May 2014 to May 2017. The inclusion criteria were age $50-75$ years, BMI $24-30$, being clinically healthy (asymptomatic). The exclusion criteria were the following: a history of gastrointestinal disease, diabetes, food allergy, use of any antimicrobial agent or probiotics or an acute infection within the preceding 2 months; use of any regular concomitant medication including any non-steroidal anti-inflammatory drugs and antioxidant products or probiotics within at least the preceding 2 weeks; pregnancy or breastfeeding; any serious organ or systemic disease, eating disorder, extensive exercise, genetic hyperlipidemia, drug or alcohol abuse, smoking, active weight loss $>5 \mathrm{~kg}$ in prior 3 months; participation in other studies within the last 30 days/ during the study and no wish to participate. The blood samples were collected at Tartu University Clinics.

From all the inclusion criteria we primarily targeted on the possible influence of RAC on blood sugar metabolism (HbA1c \%) and thus it was needed to keep this parameter equal in groups at the beginning of the study. After establishing that the inclusion/exclusion criteria were fulfilled the HbAlc was measured (2 weeks before the actual intervention/test period started) and the five participants with pre-diabetes diagnostic criteria HbAlc value 5.7-6.4 were included into the study. According to literature, the screening to detect prediabetes and diabetes enables early prevention and intervention in asymptomatic, undiagnosed adults in the USA who could be detected with pre-diabetes using the ADA guidelines. $\mathrm{HbA1c} \%$ is accepted as a good predictor for developing diabetes and according to the guidelines diabetes was defined as $\mathrm{HbAlc} \% \geq 6.5$ and pre-diabetes was defined as $\mathrm{HbA} 1 \mathrm{c} \% \geq 5.7$ [15].The participants (5 persons) were asked not to change diet habits during the investigation period (it was also checked repeatedly) and continue the previous study protocol[1] for 3 years. This study was carried out in accordance with the Declaration of Helsinki of the World Medical Association.

Innovative RAC [1] has been designed to promote cardiometabolic health (Table 1). This formulation combines pleiotropic Lactobacillus fermentum ME-3 (LFME3, deposited in the Deutsche Sammlung von Mikroorganismen und Zellkulturen, GMBH; the registration number DSM 14241) with other functional ingredients that have been used in food supplements due to different protective cardio-metabolic effects [16].
Table 1. The composition of the RAC capsule

\begin{tabular}{|l|c|c|}
\hline \multicolumn{1}{|c|}{ Ingredients } & $\begin{array}{c}\text { Quantity per recommended } \\
\text { daily dose (2 capsules) }\end{array}$ & $\begin{array}{c}\text { \% of } \\
\text { RDA }\end{array}$ \\
\hline Red Yeast rice & $\begin{array}{c}666 \mathrm{mg} \\
(10 \mathrm{mg} \text { monacolin } \mathrm{K})\end{array}$ & - \\
\hline Lactobacillus fermentum ME-3 & $60 \mathrm{mg}$ & - \\
\hline (equals 6 $\left.\times 10^{9} \mathrm{LAB}\right)$ & - \\
\hline Lbiquinol (Kaneka QH ${ }^{\mathrm{TM}}$ ) & $30 \mathrm{mg}$ & $83 \%$ \\
\hline Vitamin E & $30 \mathrm{mg}$ & $60 \%$ \\
\hline Vitamin B1 & $10 \mathrm{mg}$ & $72 \%$ \\
\hline Vitamin B6 & $0.66 \mathrm{mg}$ & $50 \%$ \\
\hline Vitamin B9 & $1 \mathrm{mg}$ & $60 \%$ \\
\hline $\begin{array}{l}\text { Vitamin B12 } \\
\text { Other ingredients: maltodextrin (filling agent), magnesium salts of fatty acids (anti- } \\
\text { caking agent), silica dioxide (anti-aggregating agent), vegetable capsule (hydroxyl- } \\
\text { propyl-methyl-cellulose), soy lecithin }\end{array}$ & $100 \mu \mathrm{g}$ & \\
\hline
\end{tabular}

To measure oxLDL the immunoassay ELISA kit (Mercodia AB, Uppsala, Sweden) was used. Analyses of metabolic indices (plasma glucose and lipids: total cholesterol Tcholesterol, LDL-cholesterol, HDL-cholesterol, TG), HbA1c and homocysteine (Hcy) were performed with standard laboratory methods using certified assays in the United Laboratories of the Tartu University Hospital, Estonia. Intervals for routine laboratory tests proposed by the Nordic Reference Interval Project [17] were used as references.

\section{Statistical Analysis}

Calculations were performed using commercially available statistical software packages (Statistics for Windows and Graph Pad PRISM Version 2.0). All values are given as mean and standard deviation (mean $\pm \mathrm{SD}$ ). Statistically significant differences between the different values of each asymptomatic patient during the period of 3 years of consumption of LFME-3 containing capsules were determined by using Student's t-test. In all analyses, p-values $<0.05$ were considered to be statistically significant.

\section{Results}

During consumption of RAC capsules for 3 years (two capsules per day) participants did not have any complaints and had statistically significant positive cardio-metabolic shifts (Table 2). The most impressive change appeared in the decrease of LDL-chlesterol level. HbA1c, oxLDL, TG/HDL-chlesterol ratio and Hcy also declined significantly whereas HDL-chlesterol level increased. Administration of LFME-3 containing RAC leads to significant decline of the level of $\mathrm{HbAlc} \%$ after 3 year consumption (Table 2). The use of RAC also declined the insulin resistance marker the TG/HDL-chlesterol ratio (Table 2) which correlates inversely with the plasma level of small dense LDL particles. The ratio TG/HDL-chlesterol shows promise as an index of the atherogenic properties of the plasma lipid profile and reflects the INS resistance (Table 2) $[5,16]$. 
Tiiu Kullisaar (2019) The Reg 'Activ Cholesterol Positive Effects in Asymptomatic Volunteers with Borderline Values of Cardio-Metabolic Risk Factors are Sustainable in Long-Term Run

Table 2. Markers for cholesterol fractions, triglycerides, prediabetes, inflammation, oxLDL at 0 week (baseline) and after consumption of probiotic LFME-3 containing RAC (2 capsules/per day, 3 years)

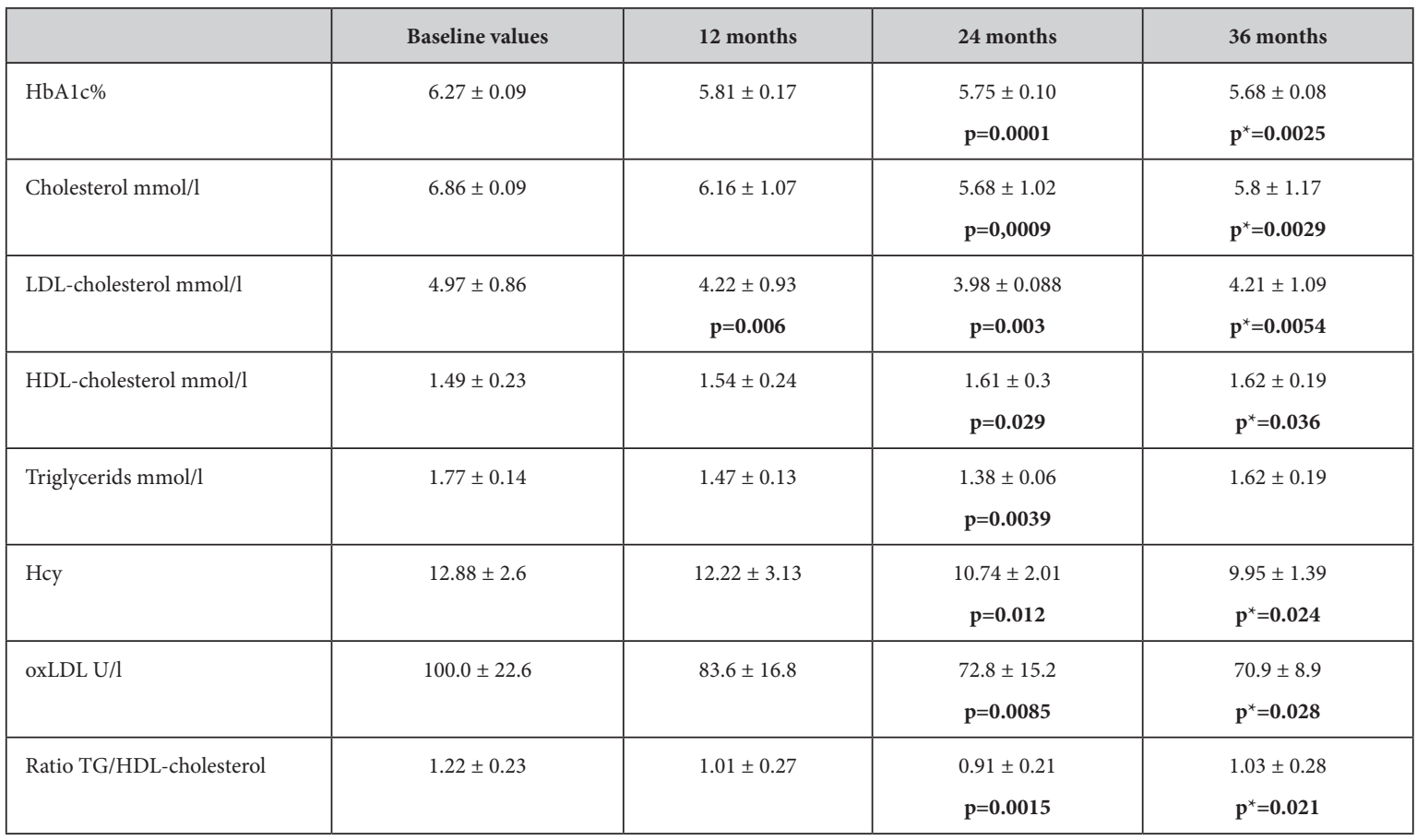

$\mathrm{p}=$ baseline values $/ 24$ months; $\mathrm{p}^{*}=$ baseline values $/ 36$ months.

\section{Discussion}

The pathogenesis of diabetes and CVD has several overlapping cardiometabolic signs. Within the framework of a double-blond, placebo controlled study clinical trial (ISRCTN55339917) we aimed to do a very long clinical sub-trial. During such a long-term study extremely important is careful fulfilment all rules (no do change typical diet habits, etc). To be convinced that such complicated options were actually followed during such long period the number of participants was limited, so it was possible to check very frequently real following of all needed options by participants. The special accent was focused on $\mathrm{HbAlc}$. One of the reasons was the information that despite positive treatment effects of so called "aggressive statins" there are several sideeffects concerns, including problems with blood sugar-related aspects. The RAC contains monacolin K (so called "mild statin"), which mechanism of action is similar to statins, however the level of HbA1c decreased during the period of 3 years. Innovative composition of RAC showed also some other beneficial effects, as a decline of levels of LDL-chlesterol, oxLDL, Hcy and TG/HDL-chlesterol ratio. It should be mentioned that Hcy is also one of the risk factor of cardiometabolic diseases [18]. Obviously, one of components in RAC, a pleiotropic probiotic LFME3 has an independent substantial role regarding mentioned beneficial effects.

Human health is closely linked to and dependent on the quality and variety of gut microbiota. The complex ecosystem inside the gut consists of complex interrelations between the host, nutrients, microbiological and environmental factors - and we are just beginning to understand the mechanisms $[6,19-21]$. It has been established in several settings by different investigators that the effect of probiotics on lipid metabolism parameters of the host can be quite strain and host specific [22-23]. The anti-oxidative and anti-atherogenic effects of LFME- 3 have been tested for in several previous experiments in vitro, in animal models and human trials. The tested media containing the strain have been goat milk, cheese, yoghurt, kefir and capsules [8, $11,12,14]$.

In our previous studies all subjects in the LFME-3 enriched kefir group harbored lactobacilli during the trial [14].

It has been established that probiotics may bind bile acids and thus remove them from the enterohepatic circulation thus favoring the decline of Cholesterol level [24]. There may exist other possible mechanisms: from previous studies it is known that LFME-3 has good activity of glycosyl-hydrolases like alpha-galactosidase, beta-galactosidase, alpha-glycosidase, beta-glycosidase and betaglycoronidase [1, 24]. Glycosyl-hydrolases are mucin degrading enzymes and the mucin degradation by intestinal bacteria and its use as a carbon source stimulate goblet cells to increase mucus production [24-25]. The effects of decrease of LDL level with normal and borderline high values may be associated with the assimilation of Cholesterol during bacterial multiplication or binding Cholesterol to the surface of the cell wall of bacteria, and this way preventing the absorption of Cholesterol from the intestine into the bloodstream [26].

The production of Short Chain Fatty Acids (SCFA) in the gut could lead to suppression of the synthesis of fatty acids in the liver, thus decreasing the TG and VLDL-cholesterol levels secretion rate, also stimulate carbohydrate oxidation, improved glucose tolerance and reduce the levels of some pro-inflammatory cytokines [27-28].

Elevated OxS increases the production of oxLDL particles that are less likely to be recognizable to LDL receptors. The higher the LDL 
content the greater the possibility of production of oxLDL. Uptake of oxLDL particles by arterial wall macrophages leads to the formation of foam cells under the endothelium and atherosclerosis. The consumption of RAC capsules for 3 years decreased both the amounts of LDL and oxLDL, while the amount of HDL-Cholesterol increased. The effect of monacolin K and LFME-3 may be the reasons for that [29-30].

The possible mechanisms of action of homocystein (Hcy) in contribution to vascular risk include endothelial dysfunction, inflammatory response, oxidation of LDL-cholesterol, and platelet activation [18]. The level of Hcy declined in RAC users.

The type 2 diabetes is a disease with multi-factorial etiology that is characterized by hyperglycemia due to resistance to insulin signal and insufficient compensatory response in secretion of this hormone. Insulin resistance is associated with cardiovascular disease pathogenesis [27]. Colonization with LFME-3 was negatively associated with blood glucose level thus showing its possible potential for carbohydrate metabolism modification in the gut or limiting their absorbance into the blood. In recent scientific papers it is shown that gastrointestinal $\mathrm{OxS}$ is associated with the non-specific glycation, which in turn may influence the long term level of blood sugar. The ability of LFME-3 to decrease the level of $\mathrm{HbAlc} \%$ was demonstrated in our study. The up-regulation of pro-inflammatory cytokines associated with $\mathrm{OxS}$ and inflammation as well as non-specific glycation may reduce glucose transporter type 4 (GLUT4) expressions and translocation to the plasma membrane in human adipocytes and muscle cells, resulting in decreased insulin-stimulated glucose uptake [28-29]. The same may happen to GLUT2, characterized with low affinity and high capacity for glucose. This isoform of glucose transporter is involved in glucosesensing in pancreatic $\beta$-cells, liver, and the hypothalamus as well as triggering the glucose mediated INS secretion cascade and/or INS synthesis. GLUT2 ensures that the glucose flux is proportional to blood glucose concentrations [30]. The RAC formula contains monacolin $\mathrm{K}$ that inhibits HMG-CoA-reductase, the key enzyme of cholesterol synthesis. This formula also contains powerful antioxidants vitamin E and ubiquinol (vitamin Q). A study by Macchi and colleagues [31] also demonstrated the beneficial effects of a probiotic, red yeast rice extract, niacin and coenzyme Q10 targeting dyslipidemia (high LDLcholesterol) in two different mechanisms, by inhibition of cholesterol synthesis and by reabsorption.

Targeting hyperglycemia and OxS simultaneously may work in cooperation in correcting the lipid profile abnormalities (e.g. elevated LDL levels) and improve OxS which increases the susceptibility of LDL particles to oxidation and glycation [32]. In turn this is an option to prevent endothelial dysfunction and atherosclerosis. Intake of cysteine is the rate-limiting factor in human glutathione (GSH) biosynthesis. GSH is a regulator of many body functions and the principal cellular antioxidant. RAC contains L-cysteine and LFME-3 can transport and synthesize glutathione and has the ability of redox cycling of glutathione $[10,14,33]$. Vitamins B1, B6, B9 and B12 in the RAC composition have several cardio-metabolic effects including the control of Hcy level. Probably the simultaneous targeting of hyperglycemia and OxS could be at least as effective as intensive treatment of hyperglycemia in prevention of T2DM complications [33]. As CVD and T2DM are chronic conditions needing an (almost) lifelong attention and management there is a place for food supplements and functional foods in the complex management of them [34-35].

\section{Conclusion}

In recent through meta-analysis was concluded that probiotics may cause beneficial shift in blood lipid profile and fast blood glucose in patients with T2DM. We have repeatedly showed that antioxidative LFME3 as several beneficial cardio-metabolic effects. Now we established that the RAC, comprising also LFME3, has positive effects in asymptomatic volunteers with borderline values of risk factors in very long run. A reduction in OxS status and simultaneous control of other cardio-metabolic risk factors seems to be a good option to improve prevention and treatment strategies. As CVD and T2DM are chronic conditions requiring a life-long attention and management an implementation of some adjuvant components (eg. nutraceuticals), with verified effects, and deserved attention regarding more comprehensive management.

\section{Acknowledgement}

The author would like to thank the participants who participated in the present study. The author wish to express appreciation to professor Mihkel Zilmer for the valuble theoretical advices and associated professor Dr. Aune Rehema for critical editing of English grammar and syntax of the manuscript. We thank the VF Bioscience Company for donating the RAC capsules used in this work.

\section{References}

1. Kullisaar T, Zilmer K, Salum T, Rehema A, Zilmer M (2016) The use of probiotic L. fermentum ME-3 containing Reg'Activ Cholesterol supplement for 4 weeks has a positive influence on blood lipoprotein profiles and inflammatory cytokines: an open-label preliminary study. Nutr J 28: 1-6.

2. Parthasarathy S, Raghavamenon A, Garelnabi MO, Santanam N (2010) Oxidized low-density lipoprotein. Methods Mol Biol 610: 403-417. [crossref]

3. Antonelli M, Kushner I (2017) It's time to redefine inflammation. FASEB $J$ 31: 1787-1791. [crossref]

4. Pitsavos C, Tampourlou M, Panagiotakis DB, Skoumas Y, Chrysohoou C, et al (2007) Association Between Low-Grade Systemic Inflammation and Type 2 Diabetes Mellitus Among Men and Women from the ATTICA Study. Rev Diabet Stud 4: 98-104.

5. BalÃ $\mu \mathrm{t} \AA_{j}$ ev R, Koido K, Vasar V, Janno S, Kriisa K, et al. (2017) Inflammatory, cardio-metabolic and diabetic profiling of chronic schizophrenia. Eur Psychiatry 39: $1-10$. [crossref]

6. Chistiakov DA, Bobryshev YV, Kozarov E, Sobenin IA, Orekhov AN (2015) Role of gut microbiota in the modulation of atherosclerosis-associated immune response. Front Microbiol 6: 671. [crossref]

7. Mikelsaar M, Annuk H, Stsepetova J, Mändar R, Sepp E, et al. (2002) Intestinal lactobacilli of Estonian and Swedish children. Microb Ecol Health Dis 14: 75-80.

8. Kullisaar T, Zilmer M, Mikelsaar M, Vihalemm T, Annuk H, et al. (2002) Two antioxidative lactobacilli strains as promising probiotics. Int J Food Microbiol 72 : 215-224.

9. Annuk H, Shchepetova J, Kullisaar T, Songisepp E, Zilmer M, et al. (2003) Characterization of intestinal lactobacilli as putative probiotic candidates. $J$ Appl Microbiol 94: 403-412. [crossref]

10. Kullisaar T, Songisepp E, Aunapuu M, Kilk K, Arend A, et al. (2010) Complete glutathione system in probiotic Lactobacillus fermentum ME-3. Prikl Biokhim Mikrobiol 46: 527-531. [crossref]

11. Kullisaar T, Songisepp E, Mikelsaar M, Zilmer K, Vihalemm T, et al. (2003) Antioxidative probiotic fermented goats' milk decreases oxidative stress-mediated atherogenicity in human subjects. Br J Nutr 90: 449-456.

12. Songisepp E, Kals J, Kullisaar T, Mändar R, Hütt P, et al. (2005) Evaluation of the functional efficacy of an antioxidative probiotic in healthy volunteers. Nutr J 4: 22 . 
Tiiu Kullisaar (2019) The Reg 'Activ Cholesterol Positive Effects in Asymptomatic Volunteers with Borderline Values of Cardio-Metabolic Risk Factors are Sustainable in Long-Term Run

13. Kaur S, Kullisaar T, Mikelsaar M, Eisen M, Rehema A, et al. (2008) Successful management of mild atopic dermatitis in adults with probiotics and emollients. Central European Journal of Medicine 3: 215-220.

14. Kullisaar T, Shepetova J, Zilmer K, Songisepp E, Rehema A, et al. (2011) An antioxidant probiotic reduces postprandial lipemia and oxidative stress. Centr Eur $J$ Biol 6: 32-40.

15. American Diabetes Association (2014) Diagnosis and classification of diabetes mellitus. Diabetes Care 37: S81-S90.

16. Murguia-Romero M, Jimenez-Flores JR, Sigrist-Flores SC, Espinoza-Camacho MA, Jimenez-Morales M, Pina E, et al. (2013) Plasma triglyceride/HDL-cholesterol ratio, insulin resistance, and cardio-metabolic risk in young adults. $J$ Lipid Res 54: 2795-2799.

17. Rustad P, Felding P, Franzon L, Kairisto V, Lahti A, et al. (2004) The Nordic Reference Interval Project 2000: recommended reference intervals for 25 common biochemical properties. Scand J Clin Lab Invest 64: 271-284.

18. Unt E, Zilmer K, Mägi A, Kullisaar T, Kairane C, et al. (2008) Homocysteine status in former top-level male athletes: possible effect of physical activity and physical fitness. Scand J Med Sci Sports 18: 360-366.

19. Canny GO, McCormick BA (2008) Bacteria in the intestine, helpful residents or enemies from within? Infect Immun 76: 3360-3373. [crossref]

20. Cani PD, Delzenne NM (2009) The role of the gut microbiota in energy metabolism and metabolic disease. Curr Pharm Des 15: 1546-1558. [crossref]

21. Sommer F, Bäckhed F (2013) The gut microbiota--masters of host development and physiology. Nat Rev Microbiol 11: 227-238. [crossref]

22. Kekkonen RA, Lummela N, Karjalainen H, Latvala S, Tynkkynen S, et al. (2008) Probiotic intervention has strain-specific anti-inflammatory effects in healthy adults. World J Gastroenterol 14: 2029-2036. [crossref]

23. Sanaie S, Ebrahimi-Mameghani M, Mahmoodpoor A, Shadvar K, Golzari SE (2013) Effect of a Probiotic Preparation (VSL\#3) on CardiovascularRisk Parameters in Critically-Ill Patients. J Cardiovasc Thorac Res 5: 67-70. [crossref]

24. Burgain J, Scher J, Francius G, Corgneau M, Revol-Junelles AM, et al. (2014) Lactic acid bacteria in dairy food: surface characterization and interactions with food matrix components. Adv Colloid Interface Sci. 213: 21-35.

25. Papadimitriou K, Zoumpopoulou G, Foligné B, Alexandraki V, Kazou M, et al. (2015) Discovering probiotic microorganisms: in vitro, in vivo, genetic and omics approaches. Front Microbiol 6: 58
26. Bernini LJ, Simao ANC, Alfieri DF, Lozovoy MAB, Mari NL, et al. (2016) Beneficial effects of Bifidobacterium lactis on lipid profile and cytokines in patients with metabolic syndrome: A randomized trial. Effects of probiotics on metabolic syndrome. Nutrition 32: 716-719.

27. Asemi Z, Alizadeh SA, Ahmad K, Goli M, Esmaillzadeh A (2016) Effects of beta-carotene fortified synbiotic food on metabolic control of patients with type 2 diabetes mellitus: A double-blind randomized cross-over controlled clinical trial. Clin Nutr $35: 819-825$.

28. Shirouchi B, Nagao K, Umegatani M, Shiraishi A, Morita Y, et al. (2016) Probiotic Lactobacillus gasseri SBT2055 improves glucose tolerance and reduces body weight gain in rats by stimulating energy expenditure. Br J Nutr 116: 451-458. [crossref]

29. EFSA (2011) Scientific Opinion on the substantiation of health claims related to monacolin K from red yeast rice and maintenance of normal blood LDL cholesterol concentrations (ID 1648, 1700) pursuant to Article 13(1) of Regulation (EC) No 1924/2006. EFSA J 9: 2304.

30. Verhoeven V, Hartmann ML, Remmen R, Wens J, Apers S, et al. (2013) Red yeast rice lowers cholesterol in physicians - a double blind, placebo controlled randomized trial. BMC Complement Altern Med 13: 178.

31. Macchi C, Botla M, Bosisio R (2017) Efficasy and safety of a nutraceutical with probiotic and red yeast rice extract in patients with moderaate hypercholesterolemia: randomized, double-blind, placebo-controlled study. European Atherosclerosis Society Annual Congress; Prague, Czech Republic. Abstract78448.

32. Erejuwa OO, Sulaiman SA, Wahab MS, Sirajudeen KN, Salleh MS, et al. (2011) Glibenclamide or metformin combined with honey improves glycemic control in streptozotocin-induced diabetic rats. Int J Biol Sci 7: 244-252. [crossref]

33. Rehema A, Kullisaar T, Seer K, Reinmann K, et al. (2015) Proteomic proof that a probiotic elevates glutathione level in human serum. Open Life Sci 10: 2391-5412.

34. Chen G, Wang H, Zhang X, Yang ST (2014) Nutraceuticals and functional foods in the management of hyperlipidemia. Crit Rev Food Sci Nutr 54: 1180-1201. [crossref]

35. Guo Z, Liu XM, Zhang QX, Shen Z, Tian FW, et al. (2011) Influence of consumption of probiotics on the plasma lipid profile: a meta-analysis of randomised controlled trials. Nutr Metab Cardiovasc Dis 21: 844-850.

\section{Citation:}

Tiiu Kullisaar (2019) The Reg Activ Cholesterol Positive Effects in Asymptomatic Volunteers with Borderline Values of Cardio-Metabolic Risk Factors are Sustainable in Long-Term Run. Endocrinol Diabetes Metab J Volume 3(2): 1-5. 